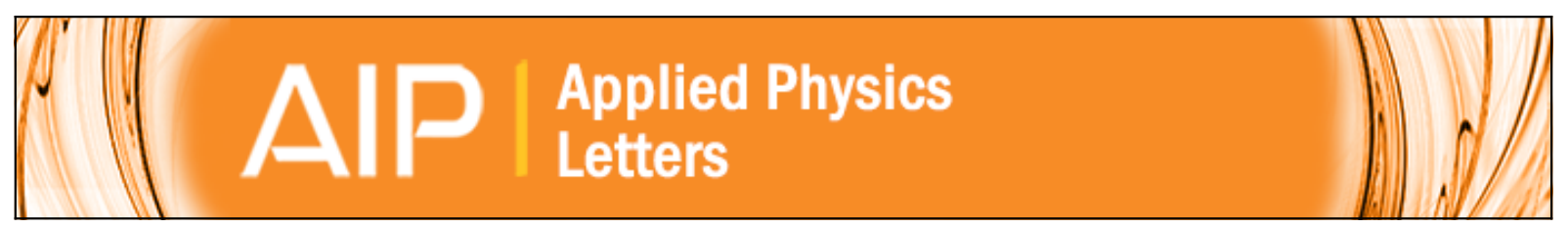

\title{
Direct manipulation of the uncompensated antiferromagnetic spins in exchange coupled system by $\mathrm{GeV}$ ion irradiation
}

Amitesh Paul, N. Paul, C. Trautmann, S. Mattauch, Jaru Jutimoosik, Rattikorn Yimnirun, Saroj Rujirawat, Britta Höpfner, Iver Lauermann, M. Lux-Steiner, and P. Böni

Citation: Applied Physics Letters 100, 253102 (2012); doi: 10.1063/1.4729472

View online: http://dx.doi.org/10.1063/1.4729472

View Table of Contents: http://scitation.aip.org/content/aip/journal/apl/100/25?ver=pdfcov

Published by the AIP Publishing

\section{Articles you may be interested in}

Enhancement of the magnetic interfacial exchange energy at a specific interface in $\mathrm{NiFe} / \mathrm{CoO} / \mathrm{Co}$ trilayer thin films via ion-beam modification

J. Appl. Phys. 115, 073901 (2014); 10.1063/1.4865569

Magnetic coercivity of focused ion beam irradiated lines in a $\mathrm{Pt} / \mathrm{Co}(1.4 \mathrm{~nm}) / \mathrm{Pt}$ film

J. Appl. Phys. 109, 093919 (2011); 10.1063/1.3580506

Exchange shift of stripe domains in antiferromagnetically coupled multilayers

Appl. Phys. Lett. 91, 132507 (2007); 10.1063/1.2793626

Magnetization reversal, asymmetry, and role of uncompensated spins in perpendicular exchange coupled systems

Appl. Phys. Lett. 89, 232507 (2006); 10.1063/1.2402882

Spin wave frequency shifts in exchange coupled ferromagnet/antiferromagnet structures: Application to $\mathrm{Co} / \mathrm{CoO}$ J. Appl. Phys. 81, 4485 (1997); 10.1063/1.364986

\section{A.P $\left.\right|_{\text {Applied Physics }} ^{\text {Journal of }}$}

Journal of Applied Physics is pleased to announce André Anders as its new Editor-in-Chief 


\title{
Direct manipulation of the uncompensated antiferromagnetic spins in exchange coupled system by $\mathrm{GeV}$ ion irradiation
}

\author{
Amitesh Paul, ${ }^{1, a)}$ N. Paul, ${ }^{2}$ C. Trautmann, ${ }^{3}$ S. Mattauch, ${ }^{4}$ Jaru Jutimoosik, ${ }^{5}$ \\ Rattikorn Yimnirun, ${ }^{5}$ Saroj Rujirawat, ${ }^{5}$ Britta Höpfner, ${ }^{2}$ Iver Lauermann, ${ }^{2}$ \\ M. Lux-Steiner, ${ }^{2}$ and P. Böni ${ }^{1}$ \\ ${ }^{1}$ Technische Universität München Physik Department E21, James-Franck-Strasse 1, D-85748 Garching b. \\ München, Germany \\ ${ }^{2}$ Helmholtz-Zentrum Berlin für Materialien und Energie GmbH, Hahn-Meitner-Platz 1, D-14109 Berlin, \\ Germany \\ ${ }^{3}$ GSI Helmholtzzentrum für Schwerionenforschung GmbH, Planckstrasse 1, D-64291 Darmstadt, Germany \\ ${ }^{4}$ Jülich Centre for Neutrom Science Forschungszentrum Jülich GmbH, Außenstelle am FRM-II clo TU \\ München, Lichtenbergstraße 1, D-85747 Garching b. München, Germany \\ ${ }^{5}$ School of Physics, Institute of Science, Suranaree University of Technology and Synchrotron Light Research \\ Institute, Nakhon Ratchasima, Thailand
}

(Received 15 February 2012; accepted 29 May 2012; published online 18 June 2012)

\begin{abstract}
Incident ion energy to matrix electrons of a material is dissipated within a narrow cylinder surrounding the swift heavy ion path. The temperature of the lattice exceeds the melting point and upon quenching causes nanometric modifications. We present here a unique ex situ approach in manipulating the uncompensated spins in antiferromagnetic layers of ferro-/antiferromagnetic exchange coupled systems on a nanometric scale. We use the impact of relativistic heavy ion $(1-2 \mathrm{GeV})$ irradiation on such systems. We find an increase in the bias field and a restoration of the reversal via domain nucleation in the trained state. These are identified as plausible results of ion-induced antiferromagnetic ordering with little or no effect on the layer structure. This study demonstrates, therefore, the possibility of nanoscale tailoring of exchange coupled systems that survive even in the trained state. C 2012 American Institute of Physics. [http://dx.doi.org/10.1063/1.4729472]
\end{abstract}

The passage of ions through matter has an interaction time $\sim 10^{-17}$ s (e.g., a $5 \mathrm{MeV} /$ nucleon ion has a velocity $\approx 10 \%$ of the speed of light) and can create atomic point or extended defects within a hot cylindrical zone ( $\sim 10 \mathrm{~nm}$ radius) of atoms in quasi-thermal equilibrium. ${ }^{1}$ The slowing down of ions results from energy loss in solids. This can be either by inelastic collisions of the ions with electrons or by elastic collisions with the nuclei of the target atoms. A simple choice of the ion energies determines if it is in the regime of collision cascades or of electronic excitation with subsequent electron-phonon coupling. $S_{\mathrm{n}}$ $[\mathrm{keV} / \mathrm{nm}]$ is the nuclear stopping power that dominates for the $\mathrm{keV}$ range of ions whereas $S_{\mathrm{e}}[\mathrm{keV} / \mathrm{nm}]$ is the electronic stopping power that dominates for the $\mathrm{MeV}$ range of ions.

It has been shown earlier that the coercive field and magnetic anisotropy of magnetic multilayers (MLs) can be accurately controlled by low energy irradiation in the elastic ${ }^{2}$ or by high energy ions in the inelastic ${ }^{3}$ regime. Particularly, local manipulations of magnetization in the inelastic regime are (a) confined to a few nanometers only (b) without significant modification of the interface structure, and (c) applicable for ex situ changes. Thus irradiation techniques offer means of magnetic-tailoring devices in information technology such as in exchange biased system. ${ }^{4}$

We have taken the example of ion irradiation in exchange bias systems which demands a complete understanding $^{5}$ of various other bias-related phenomena, such as coercivity enhancement, ${ }^{6,7}$ asymmetric hysteresis loops, ${ }^{8-10}$

\footnotetext{
a) Author to whom correspondence should be addressed. Electronic mail: amitesh.paul@frm2.tum.de. Tel.: 0049 89-28914717. Fax: 0049 8928914713.
}

and last but not the least the training effect. ${ }^{11}$ An important issue has been the loss of magnetic memory upon repeated field cycling in exchange biased spin-valve systems. Direct manipulation of the antiferromagnetic (AF) spins in exchange biased systems enables us not only to tune the bias field but also to have a control over the coupled magnetization reversal mechanism.

In the low-energy ion regime, ballistic mixing causes atomic rearrangements, which subsequently modify the magnetic properties. It is possible to increase or decrease exchange bias field $H_{\mathrm{eb}}$ and also change its direction by in-situ irradiation. ${ }^{12-14}$ The effect is due to orders of magnitude more energy as compared to thermal energy deposition (e.g., field cooling). ${ }^{12,15}$ High energy heavy ions are liable to produce amorphization in a perturbed cylinder along the ion path beyond a threshold of energy transfer.

In this work, we demonstrate a unique way of affecting the uncompensated AF spins of an archetypical exchange coupled system as we irradiate it with $\mathrm{GeV}$ energy ions. The irradiations are done after deposition of the films in absence of any in-situ field. We show here that the irradiated samples have an increase in their $H_{\mathrm{eb}}$. More interesting is the manipulation of the magnetization reversal mechanism that persists even in the trained state. Such unique manipulation of reversal mechanism has not been reported thus far.

We have investigated a ML of the composition $\mathrm{SiO}_{2} /[\mathrm{Co}(11.0 \mathrm{~nm}) / \mathrm{CoO}(5.0 \mathrm{~nm}) / \mathrm{Au}(22.5 \mathrm{~nm})]_{\mathrm{N}=16}$. The usual pressure in the magnetron sputtering chamber was $3 \times 10^{-3}$ mbar in $\mathrm{Ar}$ atmosphere during deposition with a base pressure of $1 \times 10^{-7}$ mbar. We employ an ultra-violet light assisted oxidation at an $\mathrm{O}_{2}$ pressure of 200 mbar at 
$50{ }^{\circ} \mathrm{C}$ for $1 \mathrm{~h}^{16}{ }^{16}$ Irradiations were performed at GSI Helmholtzzentrum für Schwerionenforschung. Four $15 \times 15 \mathrm{~mm}^{2}$ samples were cut from a single homogeneous piece $\left(30 \times 30 \mathrm{~mm}^{2}\right)$ for the irradiation. Thereby one can rule out any changes in their characteristics due to deposition conditions. The ion fluences used are $1 \times 10^{12}$, and $1 \times 10^{13}$ ions/ $\mathrm{cm}^{2}$ for $8.4 \mathrm{MeV} / \mathrm{amu}$ of $197 \mathrm{Au}^{+}(1.6 \mathrm{GeV})$ and $238 \mathrm{U}^{+}$ $(2 \mathrm{GeV})$ ions. Calculations using the transport of ions in matter (TRIM) code (J. P. Biersack TRIM91) indicate that GeV ions travel through the entire stack of our specimens.

Conventional in-plane magnetization loops are measured using a superconducting quantum interference device (SQUID) MPMS from Quantum design. Depth sensitive vector magnetometry was done using polarized neutron scattering at the TREFF reflectometer at FRM-2. We measure specular and off-specular intensities which contain significant information regarding the vertical and the lateral correlations of the layer structure and the magnetic structure. The neutron wavelength is fixed at $\lambda=4.73 \AA$. Details on the technique and a corresponding review can be found elsewhere. ${ }^{17}$ In our experiment four different cross sections can be distinguished, namely, non spin-flip (NSF) $\left(R_{++}\right.$and $\left.R_{--}\right)$and spin-flip (SF) channels $\left(R_{+-}\right.$and $\left.R_{-+}\right)$measuring the respective longitudinal and transverse component of magnetization with respect to the guiding field. We use a cooling field $H_{\mathrm{FC}}=+4.0 \mathrm{kOe}$ down to $10 \mathrm{~K}$ directed along an in-plane direction for all specimens. The K-edge Co X-ray absorption near-edge structure (XANES) measurements were performed in the fluorescent mode with a 13compoment Ge detector at the x-ray absorption spectroscopy beamline (BL-8) of the Siam Photon Source (electron energy of $1.2 \mathrm{GeV}$, beam current $80-120 \mathrm{~mA}$ ), Synchrotron Light Research Institute, Thailand. A double crystal Ge (220) monochromator was used to scan the synchrotron X-ray with a photon energy step of $0.30 \mathrm{eV}$. Additionally, at Berliner Elektronenspeicherring Gesellschaft für Synchrotronstrahlung GmbH (BESSY) in the CISSY end-station at the highflux beamline U41-PGM, the photon energy is swept through the L3 $(778 \mathrm{eV})$ and L2 $(798 \mathrm{eV})$ edges of Co. The absorption cross-section is measured by collecting the energy selective fluorescence yield using a commercial XES300 spectrometer with an energy resolution of $0.43 \mathrm{eV}$. Microstructural characterization has been done using crosssectional transmission electron microscopy (XTEM).

Figure 1(a) shows the hysteresis loops corresponding to the room temperature and the field cooled states of the as-
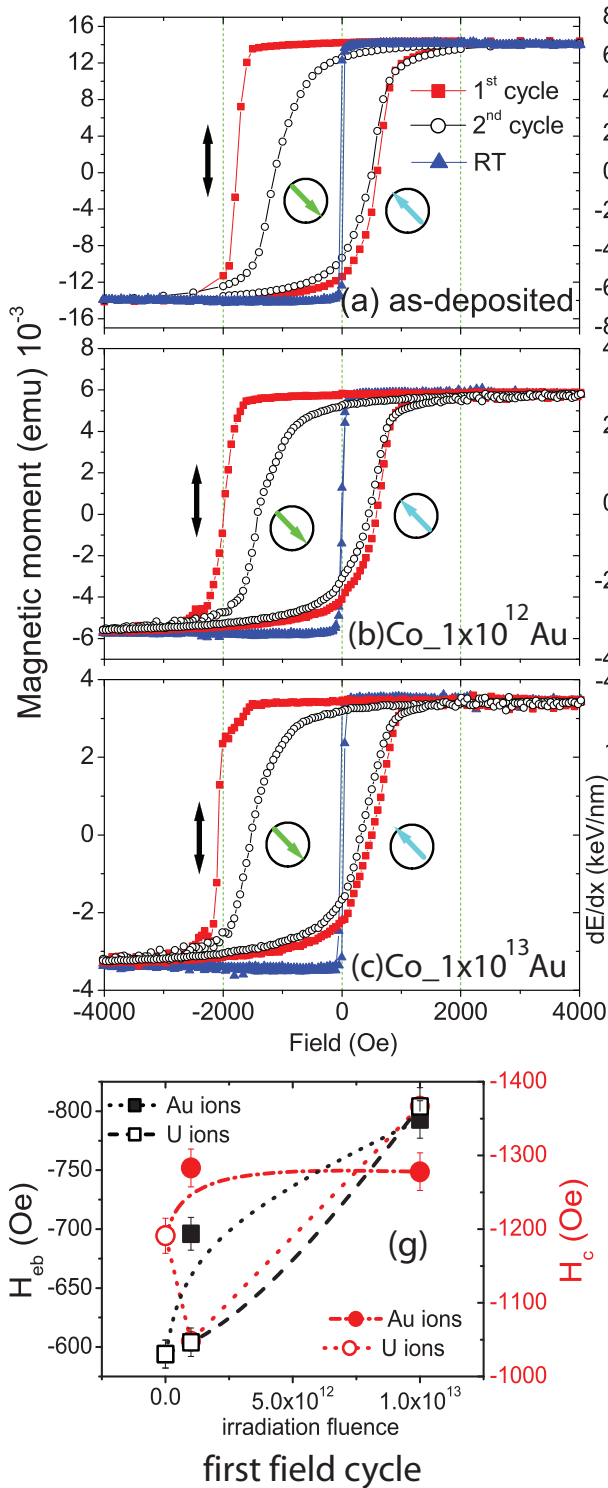
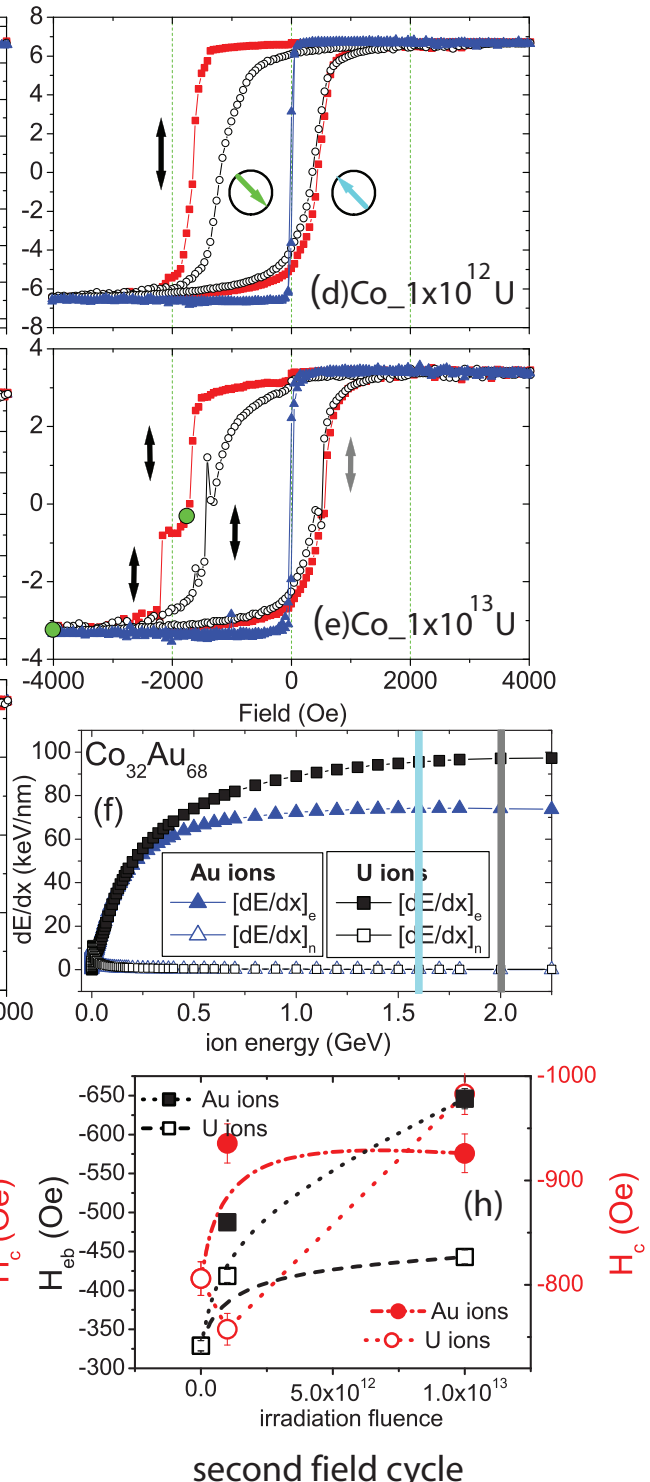

FIG. 1. SQUID magnetization hysteresis loops for the $[\mathrm{Co} / \mathrm{CoO} / \mathrm{Au}]_{16} \mathrm{ML}$ of (a) asdeposited specimen and for different fluences of (b-c) Au ion irradiation and (d), (e) $U$ ion irradiation. The measurements are done at room temperature and at $10 \mathrm{~K}$ after cooling down at $H_{\mathrm{FC}}$. The differently shaded arrows (vertical and rotating arrows) indicate the magnetization reversal mechanism along the respective branches of the loops. The green circles indicate the fields of neutron measurements shown later. (f) TRIM simulations on Co-Au target showing the electronic energy loss as compared to the nuclear energy loss with respect to the energies of $\mathrm{Au}$ and $\mathrm{U}$ ions. The ion energies used are indicated by lines. (g), (h) Plots of $H_{\mathrm{eb}}$ (squares) and $H_{\mathrm{C}}$ (circles) as a function of irradiation fluence of $\mathrm{Au}$ and $\mathrm{U}$ ions respectively during the first and second field cycles. 
deposited specimen. A characteristic negative exchange bias and an asymmetric reversal: domain wall and nucleation (nonuniform reversal) in the decreasing (applied field opposite to the cooling field) branch is indicated by vertical arrows and coherent rotation (uniform reversal) in the increasing branch indicated by rotating arrows can be seen. This asymmetry disappears, as usual, during the second field cycling with a decrease in the bias field. Figures 1(b) and 1(c) and Figures 1(d) and 1(e) show similar hysteresis loops for the Au irradiated and $U$ irradiated specimens, respectively. For the Au irradiated specimens and for the $U$ $1 \times 10^{12}$ ions irradiated specimen, even though one can see an increase in the $H_{\mathrm{eb}}$, the magnetization reversal mechanism remain quite similar to that of the as-deposited specimen. The most interesting thing happens in the magnetization reversal mechanism for the $\mathrm{U} 1 \times 10^{13}$ ions irradiated specimen. The loop is divided into two distinct parts. There are distinct steps at around 1650 and 1500 Oe (during the first and second field cycles, respectively) which are signatures of non-uniform reversal. While the upper part is fairly similar to that of the unirradiated (as-deposited) one, the lower part has undergone a significant shift. This extra shift leads to a distinct increase in exchange bias. Note that not only the first half of the first cycle (decreasing branch) shows the nonuniform reversal (indicated by vertical arrows) but also the second half of the first field cycle and also the increasing branch of the second field cycle. Henceforth we concentrate on the $\mathrm{U} 1 \times 10^{13}$ ions irradiated specimen as it is most affected by the irradiation. The $H_{\mathrm{eb}}$ and $H_{\mathrm{eb}}$ are plotted with irradiation fluence of the ions in Figure $1(\mathrm{~g})$ and $1(\mathrm{~h})$.

Controlled columnar defects produced by swift heavy ions in high temperature superconducting materials have been shown to improve their properties. ${ }^{18}$ However, in our system, GeV heavy ions could not produce any latent track damage visible by electron microscope since $\mathrm{Co} / \mathrm{Au}$ system being metallic is not a good track-recording material. Figure 2 shows the XTEM pictures for the as deposited and the sample irradiated with $1 \times 10^{13}$ ions $/ \mathrm{cm}^{2} \mathrm{U}$ ions. Interesting, however, is that the layer structure remain pretty much unaffected after irradiation only the columnar Au grains in the as-deposited sample appear fractured in the sample irradiated with U ions.

Figure 3 shows the specular reflectivity data (NSF and $\mathrm{SF}$ ) and the off-specular SF intensities corresponding to two different applied fields $\left(H_{\mathrm{a}}\right)$, indicated by circles in Figure 1(e) for the $\mathrm{U} 1 \times 10^{13}$ ions irradiated ML. We also plot the spin asymmetry (SA) signals (difference in $R_{--}$and $R_{++}$divided by the sum of the two) as we compare it with the asdeposited specimen at saturation. ${ }^{19}$ Relative variation of the multilayer Bragg peak intensities is quite evident here. Fits to the reflectivity data revealed a small increase in the $\mathrm{AF}$ layer $(\sim 1-2 \mathrm{~nm})$ at the $\mathrm{Co}-\mathrm{CoO}$ interface. The NSF intensity map shows vertically correlated multilayer interfaces added up in phase and forming the Bragg sheets in reciprocal space at the first Bragg peak position of $15 \mathrm{mrad}$. A small increase in the SF intensity at $\mathrm{Q}=0.02 \AA$, measured during the first field cycle close to the coercive field (as compared to that in saturation), is only due to an increased instability induced in the system as the layers are on the verge of flipping and cannot be attributed to coherent rotation of the layers (a similar behavior is observed during the second field cycle as well). This is further corroborated by an increase in the offspecular SF intensities near the critical edge around the coercive field, a typical signature of random non-collinear arrangement of small scale $(<1 \mu \mathrm{m})$ domains just before flipping. ${ }^{19,20}$ Distorted wave Born approximation (DWBA) has been applied in simulating the corresponding SF intensity map.

The observed increase in $H_{\mathrm{eb}}$, can be intuitively explained by intrinsic defects within the $\mathrm{Co}-\mathrm{Co}_{x} \mathrm{O}_{y}$ layers due to changes in oxidation atmosphere during the passage of high energy ions. This obviously can be related to stronger pinning of the domain walls at the defect sites. Figure 4(a) shows the measured Co K-edge XANES spectra of the samples and the reference spectra of $\mathrm{CoO}, \mathrm{Co}_{3} \mathrm{O}_{4}$, and Co metal. By considering $\mathrm{CoO}, \mathrm{Co}_{3} \mathrm{O}_{4}$, and $\mathrm{Co}$ metal as the parent components, the spectra of all the samples are fitted with varying proportion (indicated within the figure) using linear combination analysis (LCA) method in ATHENA. ${ }^{21}$ The calculated spectra using the FEFF $8.2 \operatorname{codes}^{22}$ based on ab initio multiple scattering is also compared with the measured data. A very good agreement with the measured XANES spectra in both energy positions and shapes lead us to infer that all the MLs are composed of $\mathrm{Co}$ metal and $\mathrm{CoO}$ in phaseseparated regions $\left(\mathrm{Co}_{3} \mathrm{O}_{4}\right.$ content remains similar $\left.\approx 2 \%\right)$.

We show the L-edge spectra from our U-irradiated MLs in Figure 4(b). One can see two broad peaks with broadened bases, a typical signature of localized character of the $3 \mathrm{~d}$ states. We do not observe any shift in the absorption energies as they are separated by $15.3 \mathrm{eV}$ for the MLs and also note
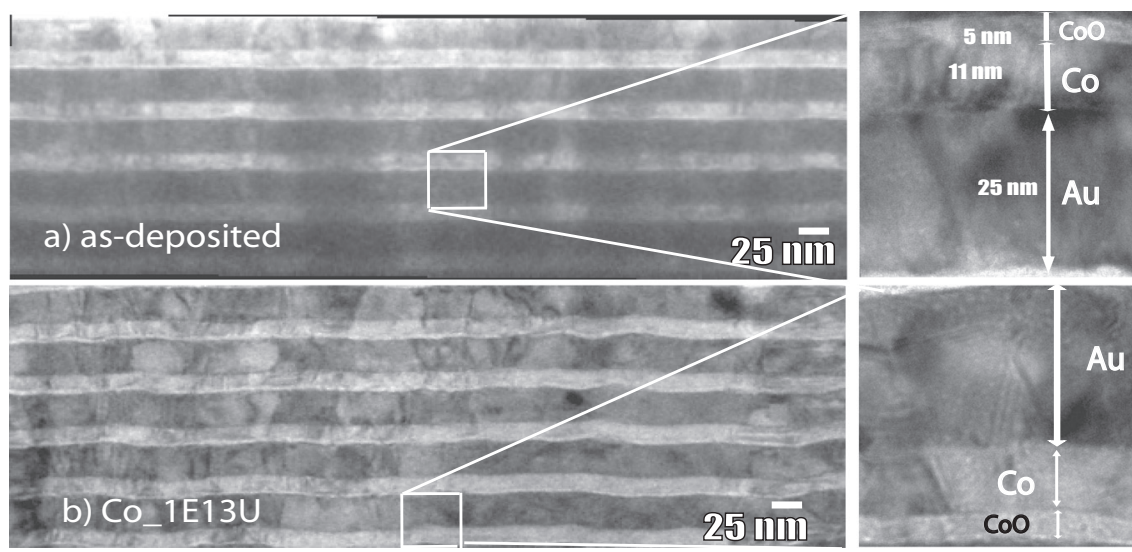

FIG. 2. XTEM micrographs of $[\mathrm{Co} / \mathrm{CoO} / \mathrm{Au}]_{16} \mathrm{ML}$ for (a) as-deposited and (b) U $1 \times 10^{13}$ ionirradiated sample. 


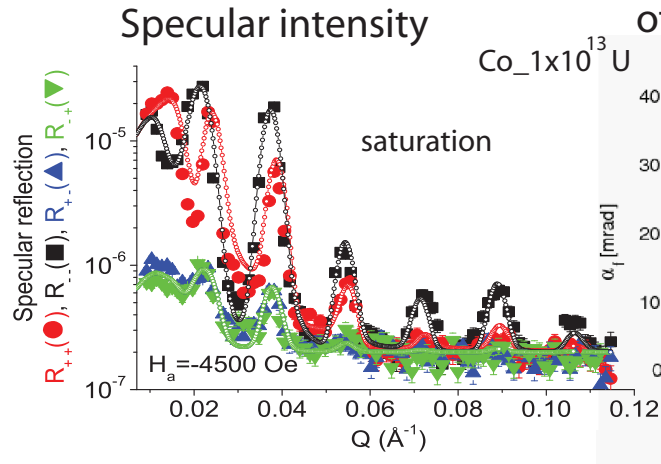

$$
\text { off-specular intensity }
$$
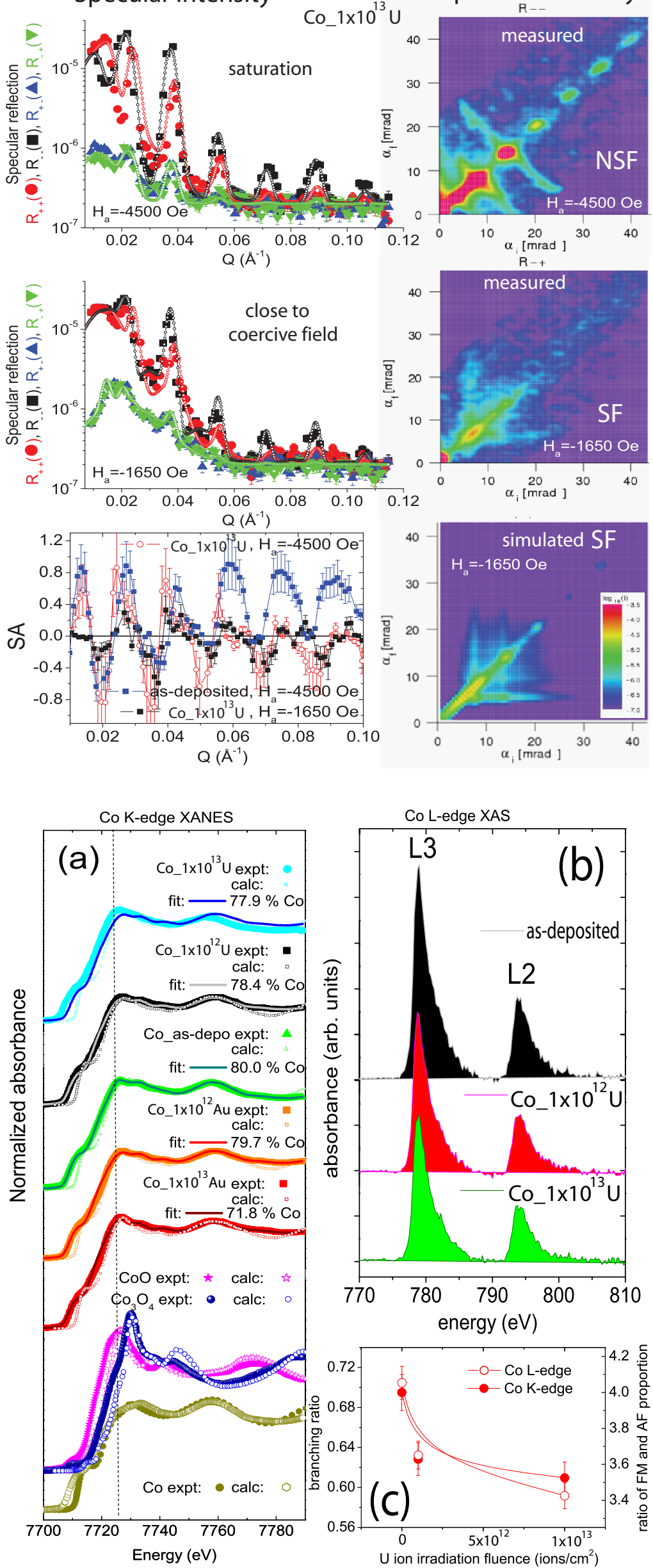

FIG. 3. Specular reflectivity patterns (solid symbols) along with their fits (open symbols) at two different applied fields, for the ML irradiated with $\mathrm{U} 1 \times 10^{13}$ ions. The measurements were done during the first field cycle at field indicated in Fig. 1(e) after cooling the sample in field down to $10 \mathrm{~K}$. $\vec{Q}=\frac{2 \pi}{\lambda}\left[\sin \left(\alpha_{\mathrm{i}}\right)+\sin \left(\alpha_{\mathrm{f}}\right)\right]$, where $\alpha_{\mathrm{i}, \mathrm{f}}$ are the incident and exit angles. The SA signal is also plotted in the bottom panel for comparison with the as-deposited specimen. The right hand panels show the measured NSF and SF intensity maps along with DWBA simulated patterns at around the coercive field for the SF channel.
FIG. 4. (a) Comparison of the measured Co K-edge normalized XANES spectra of all the MLs along with their fits and compared with the calculated spectra. Also included are the reference spectra of $\mathrm{CoO}$ and Co metal. (b) L-edge XAS spectra for the $\mathrm{U} 1 \times 10^{13}$ ions irradiated samples. (c) The intensity ratio (L-edge) and the AF-FM ratio (K-edge) for the same ML with fluence. 
that the peak-to-peak distance of the $\mathrm{L}_{2,3}$ absorption lines remains unchanged. The amount of core-hole screening by delocalized valence electrons is therefore negligible. ${ }^{23}$ To avoid the lifetime broadening and the experimental broadening contributions it is helpful to opt for the so called branching ratio calculated as $\mathrm{I}\left(\mathrm{L}_{3}\right) /\left[\mathrm{I}\left(\mathrm{L}_{2}\right)+\mathrm{I}\left(\mathrm{L}_{3}\right)\right]$. The peak intensity ratio is shown in Figure 4(c). ${ }^{24}$ The ratios are calculated from the area under the respective peaks using the IFEFFIT package. ${ }^{25}$ The changes in the intensity ratio (transfer of the spectral weight between the two edges) is a measure of the angular part of the spin-orbit operator. It shows a decreasing trend with fluence of $\mathrm{U}$ ions which further corroborates with the increasing Co valency. Therefore, the increased $H_{\mathrm{eb}}$ in our samples can be attributed to the gradual decrease in the ferromagnet (FM)-AF proportion ratio with irradiation fluence as shown in Figure 4(c). Note that our AF thickness is below a typical critical AF thickness of $\approx 10 \mathrm{~nm}^{26}$

In the electronic slowing-down regime, most of the energy of the incident ions is transferred to the host electrons, resulting in a high electronic ionization (ionic spike $\sim 10^{-14} \mathrm{~s}$ ) and/or a high temperature increase of the electronic subsystem (thermal spike $\sim 10^{-12} \mathrm{~s}$ ). Recently it has turned out that "coulomb explosion"/ion spike produces a "heat" spike and thus these are early and late aspects of the same process. According to the thermal-spike model, the energy locally deposited in matter is quickly shared among the electron gas by electron-electron interactions and then transferred to the neighboring atoms by electron-phonon (E$\mathrm{P})$ and phonon-phonon interactions. ${ }^{27}$ The temperature along the ion path can be of the order of $5 \times 10^{4} \mathrm{~K}$ within a cylindrical range of 5-10 nm along the ion path which is followed by rapid quenching $\left(10^{14} \mathrm{~K} \mathrm{~s}^{-1}\right)$ resulting in latent tracks. $S_{e}$ induced defects can be created in $\mathrm{Ti}, \mathrm{Co}$, and $\mathrm{Fe}$ as they exhibit stronger electron-phonon coupling than in $\mathrm{Ag}$, for example. The calculated threshold of defect creation induced by $S_{e}$ for Co is about $28-34 \mathrm{keVnm}^{-1}$ for $5-20 \mathrm{MeV} / \mathrm{amu}$ ions. ${ }^{28}$ Figure 1(f) shows the dominance of electronic energy loss over nuclear energy loss for $1.6 \mathrm{GeV} \mathrm{Au}$ and $2 \mathrm{GeV} \mathrm{U}$ ions in a $\mathrm{Co} / \mathrm{Au}$ system calculated by TRIM. For our system, the $S_{e}=\left.\frac{d E}{d x}\right|_{e}$ is greater than $73 \mathrm{keVnm}^{-1}$ and $97 \mathrm{keVnm}^{-1}$ for $\mathrm{Au}$ and $\mathrm{U}$, respectively ( $\propto$ ion atomic number $\mathrm{Z}^{2}$ ), which can give the latent track radius of about $\approx 2-10 \mathrm{~nm} .{ }^{3}$ The lattice temperature increase is proportional to $S_{e}$, which renders the related E-P coupling factor relatively higher for $\mathrm{U}$ ions than that for Au.

Ion-induced magnetic manipulation allows us to increase $H_{\mathrm{eb}}$ and recover untrained magnetization states in an otherwise trained exchange coupled system. The method demonstrated here is limited to nanometric scale with little influence on their interface structure. Such local manipulation holds promise for post production extrinsic treatment of integrated magnetic memory devices or sensors by choosing "specific" (energy/nucleon) ion energies.

This work was supported by the Deutsche Forschungsgemeinschaft via the Transregional Collaborative Research Centers TRR 80. We would also like to thank Ulrike Bloeck for the assistance in TEM measurements.

${ }^{1}$ B. E. Fischer and R. Spohr, Rev. Mod. Phys. 55, 907-948 (1983).

${ }^{2}$ C. Chappert, H. Bernas, J. Ferr, V. Kottler, J.-P. Jamet, Y. Chen, E. Cambril, T. Devolder, F. Rousseaux, V. Mathet, and H. Launois, Science 19, 1919 (1998)

${ }^{3}$ A. Paul and S. Mattauch, New. J. Phys. 12, 103003 (2010).

${ }^{4}$ W. H. Meiklejohn and C. P. Bean, Phys. Rev. 102, 1413 (1956).

${ }^{5}$ J. Nogués and I. K. Schuller, J. Magn. Magn. Mater. 192, 203 (1999); A.

E. Berkowitz and K. Takano, J. Magn. Magn. Mater. 200, 552 (1999).

${ }^{6}$ M. D. Stiles and R. D. McMichael, Phys. Rev. B 59, 3722 (1999).

${ }^{7}$ M. Gruyters and D. Schmitz, Phys. Rev. Lett. 100, 077205 (2008).

${ }^{8}$ M. Gierlings, M. J. Prandolini, H. Fritzsche, M. Gruyters, and D. Riegel, Phys. Rev. B 65, 092407 (2002).

${ }^{9}$ M. R. Fitzsimmons, P. Yashar, C. Leighton, I. K. Schuller, J. Nogues, C. F. Majkrzak, and J. A. Dura, Phys. Rev. Lett. 84, 3986 (2000).

${ }^{10}$ A. Paul, E. Kentzinger, U. Rücker, and Th. Brückel, Phys. Rev. B 74, 54424 (2006).

${ }^{11}$ A. Paul and S. Mattauch, Appl. Phys. Lett. 95, 092502 (2009).

${ }^{12}$ A. Ehresmann, Recent Res. Dev. Appl. Phys. 7, 401 (2004).

${ }^{13}$ D. Engel, A. Kronenberger, M. Jung, H. Schmoranzer, A. Ehresmann, A. Paetzold, and K. Roll, J. Magn. Magn. Mater. 263, 275 (2003).

${ }^{14}$ J. Fassbender, D. Ravelosona, and Y. Samson, J. Phys. D: Appl. Phys. 37, R179 (2004).

${ }^{15}$ A. Ehresmann, D. Junk, D. Engel, A. Paetzold, and K. Roll, J. Phys. D 38, 801 (2005).

${ }^{16}$ A. Paul, D. Bürgler, M. Luysberg, and P. Grünberg, Europhys. Lett. 68, 233 (2004).

${ }^{17}$ A. Paul, Pramana 78, 1 (2012).

${ }^{18}$ D. Kanjlal, Curr. Sci. 80, 25 (2001), http://www.iisc.ernet.in/currsci/ jun252001/1560.pdf.

${ }^{19}$ A. Paul, N. Paul, and S. Mattauch, New J. Phys. 13, 063008 (2011).

${ }^{20}$ A. Paul, E. Kentzinger, U. Rücker, D. Bürgler, and P. Grünberg, Phys. Rev. B 70, 224410 (2004).

${ }^{21}$ B. Ravel and M. Newville, J. Synchrotron Radiat. 12, 537 (2005).

${ }^{22}$ A. Ankudinov, B. Ravel, J. Rehr, and S. Conradson, Phys. Rev. B 58, 7565 (1998).

${ }^{23}$ J. T. Lau, J. Rittmann, V. Zamudio-Bayer, M. Vogel, K. Hirsch, Ph. Klar, F. Lofink, and T. Möller, B. v. Issendorff, Phys. Rev. Lett. 101, 153401 (2008).

${ }^{24}$ B. Thole and G. van Der Laan, Phys. Rev. B 38, 3158 (1988).

${ }^{25}$ B. Revel and M. Newville, J. Synchrotron Radiat. 12, 537 (2005).

${ }^{26}$ X. Y. Lang, W. T. Zheng, and Q. Jiang, Nanotechnology 18, 155701 (2007).

${ }^{27}$ Z. G. Wang, C. Dufour, E. Paumier, and M. Toulemonde, J. Phys.: Condens. Matter 6, 6733 (1994).

${ }^{28}$ A. Dunlop and D. Lesueur, Rad. Eff. Defects Sol. 126, 123 (1993). 